\title{
Amorphous Silicon and Carbon Nanotubes Layered Thin-Film Based Device for Temperature Sensing Application
}

\author{
Vineet Rojwal, Monoj Kumar Singha, and T. K. Mondal
}

\begin{abstract}
This paper proposes an integrated layered doped and undoped amorphous silicon thin-film based temperature sensing device. Temperature sensing performance has been measured for thin film p-i-n (p-type- intrinsicn-type) configuration-based diode. Linear dependency of voltage on the temperature for forward-biased diode at a constant bias current is demonstrated in the temperature range of $30-200{ }^{\circ} \mathrm{C}$. Further, the same device has been introduced with double-walled carbon nanotubes (DWCNTs) to improve the linearity of the sensor. Comparative performance of two configurations $p-i-n$ and $p$-i-n/DWCNTs for temperature sensing application has been studied. Moreover, this paper discussed the effect of the DWCNTs on the sensor parameters

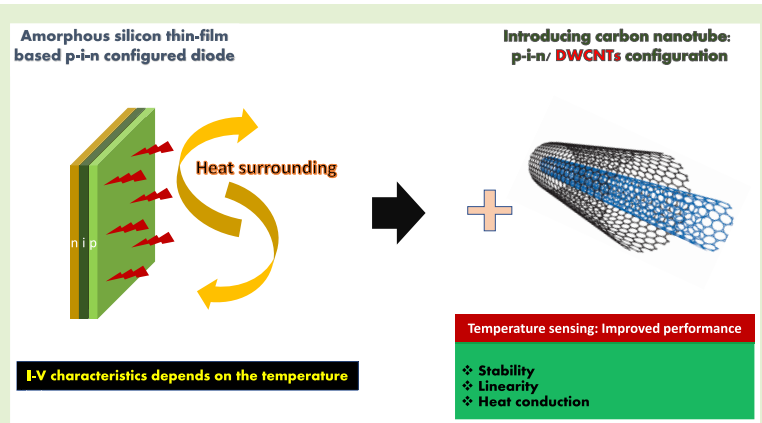
such as sensitivity, $\mathrm{S}$ and coefficient of determination, $\mathrm{R}^{2}$. The maximum sensitivity of the sensor, $22.34 \mathrm{mV} /{ }^{\circ} \mathrm{C}$ for $\mathrm{p}$-i-n configured device and $21.06 \mathrm{mV} /{ }^{\circ} \mathrm{C}$ for $\mathrm{p}$-i-n/DWCNTs configuration in a biasing current range of 10- $60 \mathrm{~mA}$ have been found. We achieved a maximum value of the coefficient of determination equal to 0.99889 for a $p$-i-n configuration and 0.99922 for a p-i-n/DWCNTs configured device.
\end{abstract}

Index Terms-Amorphous silicon, coefficient of determination, double-walled carbon nanotubes, sensitivity, temperature sensing device.

\section{INTRODUCTION}

$\mathbf{S}$ ILICON indeed is the most usable material in the electronic industry for many practical and research applications. In silicon-based technology, amorphous silicon (a-Si) has its own benefits because of the ability to be developed in thin film formation (use in less quantity of material). Amorphous silicon has been proved a very attractive thin film material for manufacturing of large surface area microelectronic and micro-photonic devices due to its optoelectronic properties. Currently, a-Si is being used in many interesting optoelectronic applications such as thin-film transistor [1], position sensor [2], temperature sensor [3], photodetector [4], colour sensor [5], images sensor [6], flat panel display [7], solar cell [8], flat panel detector [9] radiation detector [10] etc.

Temperature sensing is one of the basic optoelectronic applications at any thermal based reactor environment, and

Manuscript received August 20, 2020; accepted September 7, 2020. Date of publication September 21, 2020; date of current version January 6,2021 . The associate editor coordinating the review of this article and approving it for publication was Dr. Eui-Hyeok Yang. (Corresponding author: Vineet Rojwal.)

The authors are with the Department of Instrumentation and Applied Physics, Indian Institute of Science, Bengaluru 560012, India (e-mail: vineetrojwal@iisc.ac.in; monojsingha@iisc.ac.in).

Digital Object Identifier 10.1109/JSEN.2020.3025034 used in any specific part of the machine, furnace, wearable device etc. [11]. Considering this application, some devices are already being used like RTD (Resistance temperature detector) [12], thermocouple [13], thermistor [14] and semiconductor based temperature sensor [15] etc. Surface/ skin sensing is a convenient way to non- invasively measure the temperature at process pipelines, furnaces, tanks and chemical plants. Replacement/ repairing of RTDs or thermocouples of reactors under the insulation covering is challenging; requires highly skilled workers to do, and resulting a high cost of ownership for the end user. To eliminate these ongoing obstacles for normal skin temperature monitoring, thin film-based temperature sensors need to be developed. Previous studies have demonstrated few advanced, flexible, cost saving, ecofriendly and high performance sensors which may be suitable in such environment [16], [17].

A-Si is an important material for a semiconductor temperature sensing regarding large surface utilization. Thin film a-Si based temperature sensor is suitable with integrated circuit technology and low-cost research \& development. Moreover, previous research suggests that diode voltage linearly depends on the temperature in forward-bias condition, as a result perpetuating a high sensitivity [18], [19]. A-Si thin film can be deposited onto various substrates such as quartz, glass sheet, metal sheet/ foil, already treated silicon $(\mathrm{Si})$ wafer, plastic 
and any high mechanical flexible substrate [20]-[22]. Recent electronic research has gained much attention to develop more advance and reliable electronic devices based on organic and flexible materials [23].

In this work, doped (Boron for p-type/ Phosphorus for n-type) and undoped amorphous silicon thin films [24] based diode is used for temperature sensing application with $\mathrm{p}-\mathrm{i}-\mathrm{n}$ configuration. Later, the same diode has been modified with Double-walled carbon nanotubes (DWCNTs). The diode parameters depend on the temperature such as barrier voltage, reverse saturation current, reverse breakdown voltage and bias current. When the amorphous silicon diode with $\mathrm{p}-\mathrm{i}-\mathrm{n}$ configuration is kept forward biased, charge carriers (holes and electrons) are injected from both the regions (p-type and n-type) into the intrinsic region. This process allows the current to flow through all the layers. The generated charge carriers do not recombine immediately, but basically, as the temperature rises the concentration of the intrinsic charge carriers increases, which push FERMI level of the diode closer to the intrinsic region FERMI level [25].

For temperature sensing measurements, the diode must be forward-bias with a constant bias current over the selected temperature range. The voltage across the diode with respect to temperature has been measured. There is a drawback of this method, as applied high bias current might generate excessive heat cause excessive charge carriers and the small size devices might get damaged. Therefore, applied bias current must be optimized according to the size of the device (for higher bias current device size must be large). It is necessary to apply a lower/optimized value of bias current to remove these negative effects like parasites series resistance which is the cause of degradation in device performance (linearity or sensitivity) [26]. In some recent research, a few examples of amorphous silicon-based diode have been studied as a temperature sensor and proposed device showed excellent linearity and a sensitivity of $3.2 \mathrm{mV} /{ }^{\circ} \mathrm{C}$ [3]. From literature, the diode current $I_{D}$ is often written as in terms of voltage $V_{D}$ across the diode using well known Shockley diode equation [27]:

$$
\mathbf{I}_{\mathbf{D}}=\mathbf{I}_{\mathbf{S}}\left(\mathbf{e}^{\frac{\mathbf{q}_{\mathbf{D}}}{\eta \mathbf{k T}}}-1\right)
$$

where $\mathrm{I}_{\mathrm{S}}$ is reverse saturation current, $\mathrm{V}_{\mathrm{D}}$ is the voltage across the diode, $\boldsymbol{\eta}$ is the emission coefficient, $\mathrm{q}$ is the electron charge, $\mathrm{T}$ is the temperature of the diode and $\mathrm{k}$ is the Boltzmann constant. For the condition of $\mathrm{qV}_{\mathrm{D}} \gg \eta . \mathrm{k}$.T, the relation between the voltage of the diode and temperature can be expressed from equation (1) as:

$$
\mathbf{V}_{\mathbf{D}}=\frac{\mathbf{k T}}{\mathbf{q}} \eta \ln \left(\frac{\mathbf{I}_{\mathbf{D}}}{\mathbf{I}_{\mathbf{S}}}\right)
$$

Equation (2) expresses the linear relationship between voltage and temperature, as long as the non-linear input of the reverse saturation current $\mathrm{I}_{\mathrm{S}}$, can be considered very low with compare to $\mathrm{I}_{\mathrm{D}}$. The device measurements were performed during forward-biasing at constant bias current, where the diode voltage linearly depends on temperature [28], [29]. The sensitivity of the sensor can be measured by the following expression:

$$
\mathbf{S}=\frac{\varepsilon_{\mathbf{V}}}{\varepsilon_{\mathbf{T}}}
$$

where $\boldsymbol{\varepsilon}_{\mathbf{T}}$ is temperature resolution, measuring temperature T, $\boldsymbol{\varepsilon}_{\mathbf{V}}$ is measurement system resolution and sensor sensitivity, $S=d V / d T$.

Further analysis of sensor parameter which is the coefficient of determination, $\mathrm{R}^{2}$ has been calculated to find out the interconnection between the experimental results and their linear best-fit, $f_{\mathrm{L}}(\mathrm{T})$ [30]. The coefficient of determination is used for the statistical analysis of the sensor, which evaluates how good the model is and gives more results. In this case, measurement of $\mathrm{R}^{2}$ allows us to find out the quality of the sensor in terms of linearity, by fitting with a linear model. There would be some mismatch between calculated values of linear best-fit and experimental data, so corresponds to this, root mean square (RMS) error value $\mathrm{e}_{\mathrm{T}}\left[{ }^{\circ} \mathrm{C}\right]$ has been calculated and eventually converted into error in temperature measurement using the following equation [31]:

$$
\mathbf{e}_{\mathbf{T}}\left[{ }^{\mathbf{0}} \mathbf{C}\right]=\frac{\sqrt{\frac{\sum_{\mathbf{i}=\mathbf{1}}^{\mathbf{n}}\left(\mathbf{V}_{\mathbf{D}}\left(\mathbf{T}_{\mathbf{i}}\right)-\mathbf{f}_{\mathbf{L}}\left(\mathbf{T}_{\mathbf{i}}\right)\right)^{2}}{\mathbf{n}}}}{\mathbf{S}}
$$

where: $\mathrm{n}$ is the number of set points.

Carbon nanotubes are carbon allotropic have a unique nanostructured cylindrical structure, large in length with nanoscale diameter. All carbon nanotubes have exceptional thermal conductivity along the tube, indicating the property is known as ballistic conductivity whereas shows excellent insulating feature to the axis of the tubes laterally. These properties make carbon nanotubes an interesting material for optoelectronic applications [32]-[36]. Carbon nanotubes have exceptionally high thermal conductivity, stability, electronic property and flexibility. However, In case of SWCNTs, some physical properties are very sensitive and can be demolished easily by excessive heating, chemical modification or inducing defects [37], [38]. Flexibility and thermal conductivity are the more important property for this application. Although, use of multi-walled carbon nanotubes (MWCNTs) or double-walled carbon nanotubes (DWCNTs) can get the better of these issues due to their uniqueness than single-wall structure. Because of more layers, more thermal and mechanical stability can be obtained [39]-[41]. Moreover, the development point of view, MWCNTs and DWCNTs are better than SWCNTs to synthesizes on a large scale as it is easier, stiffer and cheaper.

The logical conclusion is that now, DWCNTs/ MWCNTs would be better options as a heat absorption layer for this application. As the number of walls increases in carbon nanotubes drop in thermal conductivity takes place. DWCNTs is being used over MWCNTs here. Eventually, DWCNTs are a synthetic mixture of both SWCNTs and MWCNTs, they have good thermal and electrical stability, and flexibility when compared to individual SWCNTs and MWCNTs [39]-[44]. Double walled carbon nanotubes were used as a heat absorption layer of the sensor surface in this application. Because 


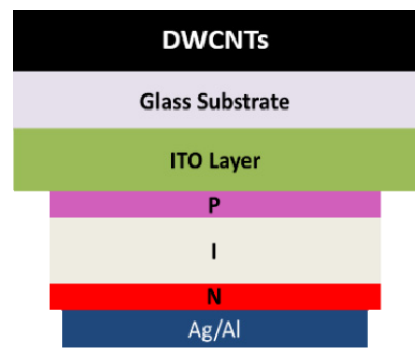

Fig. 1. Schematic diagram of amorphous silicon-based p-i-n and DWCNTs layered temperature sensing device.

of the heat from heated molecules or heated objects in the surrounding can enter into CNTs, that transfer and hold this heat at the surface of the sensing device for precise temperature measurement. It has been observed that carbon nanotubes temperature stability estimated up to $2800{ }^{\circ} \mathrm{C}$ in a vacuum and $750{ }^{\circ} \mathrm{C}$ in the air [45]-[47].

\section{EXPERIMENTAL}

All the experiments of the temperature sensor, Keithley probe station with the temperature-controlled chuck (to apply temperature at device surface) setups were performed under dark environment. Amorphous silicon-based diode as a temperature sensing device was used, which is made of doped and undoped thin film layers with p-i-n configuration. For the fabrication of the device, indium tin oxide (ITO) layer thickness of $\sim 200-250 \mathrm{~nm}$ on the glass/ quartz substrate has been deposited for the contact using the sputter deposition. Integrated layers of a-Si for n-type, intrinsic and p-type have been deposited thicknesses of $\sim 20-30 \mathrm{~nm}, \sim 300-350 \mathrm{~nm}$ and $\sim 20-30 \mathrm{~nm}$ (recipes have been optimized in the previous research [24]) respectively one after another using PECVD (plasma enhanced chemical vapor deposition) tool. After that, metal Aluminum (Al) contacts have been deposited using thermal evaporation. Furthermore, later for modification, the same device was introduced with DWCNTs (added with N, N DIMETHYL FORMAMIDE $99 \%$ ) through drop cast and spin coating (shown in Fig. 1).

For the measurements, the diode is kept at constant bias current $I_{D}$, by varying voltage $V_{D}$ across the diode. The device was placed on the surface contact of heating chuck (accuracy with $\pm 0.4{ }^{\circ} \mathrm{C}$ ) in order to monitor temperature under the dark environment and the temperature set points varied from 30 to $200{ }^{\circ} \mathrm{C}$ during measurement. By using a Keithley semiconductor device parameter analyzer, testing of the device were made for $\mathrm{I}_{\mathrm{D}}$ varied in a range from 10 to $60 \mathrm{~mA}$ (steps of $10 \mathrm{~mA}$, $\pm 50 \mathrm{pA}$ accuracy and $20 \mathrm{pA}$ resolution) and the corresponding diode voltage $V_{D}$ has been measured. The sensitivity, $S$ of the sensor was measured and calculated from $\mathrm{V}_{\mathrm{D}}$ - $\mathrm{T}$ characteristics which equivalents to temperature derivative of Equation (1). The coefficient of determination, $\mathrm{R}^{2}$ was measured at the selected bias-current range that allows us to quantify the degree of linearity of the sensor by fitting the experimental data [30]. The power dissipation $P_{D}$ of the sensor with respect to temperature in the current range of $10-60 \mathrm{~mA}$ was characterized.

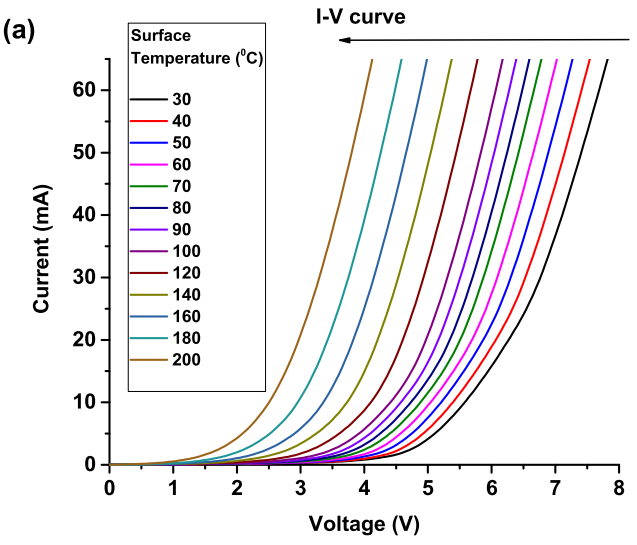

(b)
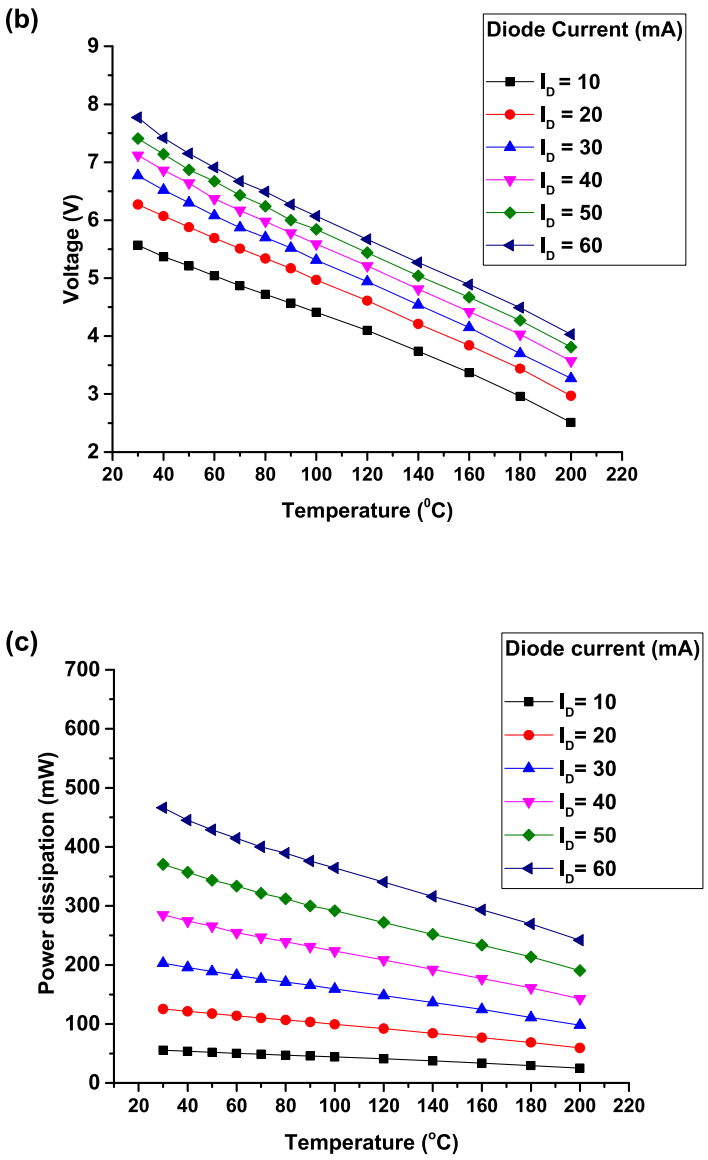

Fig. 2. (a) Current-voltage $\left(I_{D}-V_{D}\right)$ characteristics of amorphous silicon-based temperature sensor with $\mathrm{p}$-i-n/ DWCNTs configuration for temperature ranging from $30^{\circ} \mathrm{C}$ up to $200{ }^{\circ} \mathrm{C}$; (b) Measured forward voltage versus temperature $\left(V_{D}-T\right)$ curve at bias current range $\left(I_{D}=10\right.$ - $60 \mathrm{~mA}$ ); (c) Measured power dissipation versus temperature $\left(P_{D}-T\right)$ curve at same bias current range.

\section{Results and Discussion}

Fig 2(a) shows $I_{D}-V_{D}$ characteristics, for a considered temperature range of $30-200{ }^{\circ} \mathrm{C}$. Increased temperature leads to break the covalent bonds into the material that increase the number of charge carriers (both majority and minority). The built-in potential of the diode decided by FERMI level difference between $\mathrm{p}$ and $\mathrm{n}$-doped regions. 


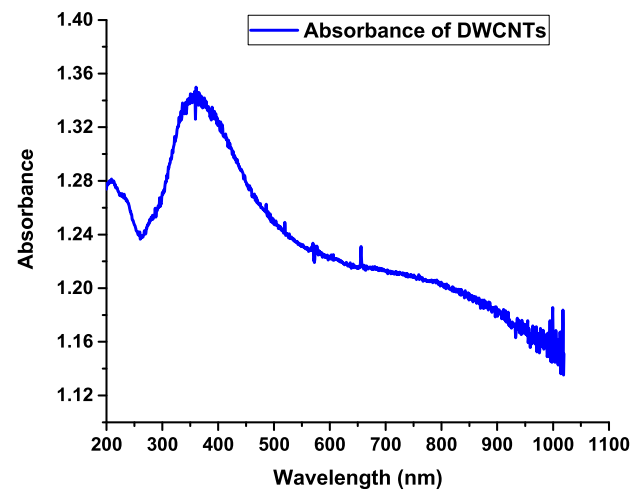

Fig. 3. Absorbance spectra of double-walled carbon nanotubes (DWCNTs) using UV-Vis-IR spectroscopy.

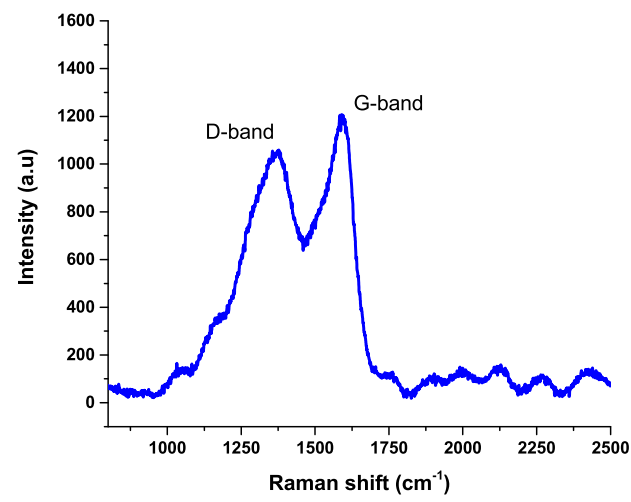

Fig. 4. Raman spectra of DWCNTs is showing D-band and G-band peaks.

As diode temperature increases, the FERMI level in both regions starts moving closer to the center of the bandgap, as a consequence, the built-in potential is decreased. From $\mathrm{I}_{\mathrm{D}}-\mathrm{V}_{\mathrm{D}}-\mathrm{T}$ characteristic exhibits a highly linear of voltage across of diode on the temperature. Result shows, the diode voltage linearly decreases with the temperature at considered constant bias-current range (10-60 mA)., shown in Fig. 2(b). Power dissipation, $\mathrm{P}_{\mathrm{D}}$ by the temperature sensor for considered current range $I_{D}=10-60 \mathrm{~mA}$ is shown in Fig 2(c). The values of $\mathrm{P}_{\mathrm{D}}$ linearly decrease with the temperature in the considered current range [26].

Absorption of double walled carbon nanotubes layer has been recorded by UV-Vis-IR spectroscopy. Before spread and coat on the sensing device, DWCNTs has been deposited on the quartz substrate (using the same recipe and timing as on device). Fig 3 shows the absorbance curve demonstrates that UV, Visible and IR photons are being absorbed by DWCNTs.

Raman scattering is an easy and quick characterization technique to characterize for all types of carbon nanotubes, particularly this technique well suited to molecular morphology of any carbon material. The Raman spectrum was recorded using LABRAM HR tool with a laser exciting beam of $532 \mathrm{~nm}$ wavelength. Since each spectrum band shows the specific frequency of vibration mode of molecules in the material. Similarly, Raman spectrum of single walled carbon nanotubes (SWCNTs) and double-walled nanotubes (DWCNTs) present three main characteristics
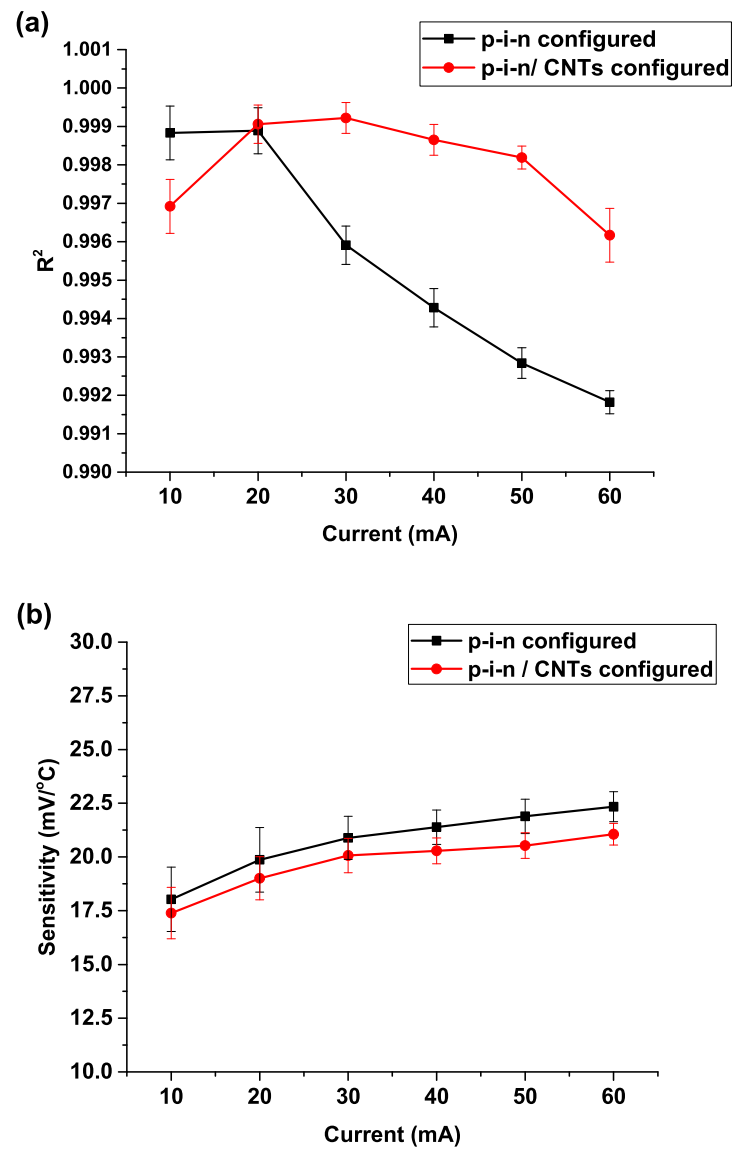

Fig. 5. Comparison of $p-i-n$ and $p-i-n / D W C N T s$ configured for bias currents between $I_{D}=10-60 \mathrm{~mA}$. (a) Coefficient of determination, $R^{2}$; (b) Sensitivity, S.

High frequency mode is associated with tangential stretching (G-band) in the range of $\sim 1500-1600 \mathrm{~cm}^{-1}$, indicates a sign of carbon nanotubes. The large D-band peak corresponds to $\sim 1350 \mathrm{~cm}^{-1}$ indicates an amorphous carbon material is allocated to a Raman scattering accompanied by double-resonance in $\mathrm{sp}^{2}$ carbon (see Fig 4) [48], [49]. Another low frequency range of Raman peaks have to be overserved in the range of $100-400 \mathrm{~cm}^{-1}$ generally known as radial breathing mode (RBM). The peak intensity of RBM seems very weak or could be rarely observed to the large diameter of nanotubes [50]-[52]. RBM is proportionate to the diameter inversely, whereas the tangential stretching (G-band) mode depends on the diameter very weakly. The defects in the walls of the nanotubes and different other carbon forms to these series of bands are still under research and not completely understood.

Furthermore, analysis of sensor parameters, the coefficient of determination, $\mathrm{R}^{2}$ and sensitivity, $\mathrm{S}$ of temperature sensor are shown in Fig 5 for the whole current range. As a result, $\mathrm{R}^{2}$ decreases with the bias current (without DWCNTs). However, in case of DWCNTs, values of $\mathrm{R}^{2}$ exhibit significantly higher and sustain, which indicates the higher linearity of the device over the considered temperature range (see Fig 5 (a)). The heat generated from heated molecules or heated objects in the surrounding can enter into DWCNTs, that transfer and hold 
TABLE I

The Sensitivity of Sensor, S And Coefficient of Determination, $R^{2}$ Calculated From $\mathbf{I}_{\mathrm{D}}-\mathbf{V}_{\mathrm{D}}-\mathbf{T}$ Characteristics of THE TEMPERATURE SENSOr For Both Configurations

\begin{tabular}{ccccc}
\hline $\begin{array}{c}\mathbf{I}_{\mathbf{p}} \\
(\mathbf{m A})\end{array}$ & $\begin{array}{c}\mathbf{S}\left(\mathbf{m V} /{ }^{\mathbf{0}} \mathbf{C}\right) \\
\text { (without DWCNTs) }\end{array}$ & $\begin{array}{c}\mathbf{R}^{\mathbf{2}} \\
\text { (without DWCNTs) }\end{array}$ & $\begin{array}{c}\mathbf{S}(\mathbf{m V} / \mathbf{0}) \\
\text { (with DWCNTs) }\end{array}$ & $\begin{array}{c}\mathbf{R}^{\mathbf{2}} \\
\text { (with DWCNTs) }\end{array}$ \\
\hline 10 & 18.03 & .99883 & 17.39 & .99692 \\
20 & 19.87 & .99889 & 19.01 & .99906 \\
30 & 20.89 & .99591 & 20.07 & .99922 \\
40 & 21.39 & .99428 & 20.29 & .99865 \\
50 & 21.89 & .99284 & 20.53 & .99819 \\
60 & 22.34 & .99182 & 21.06 & .99617 \\
\hline
\end{tabular}

(a)

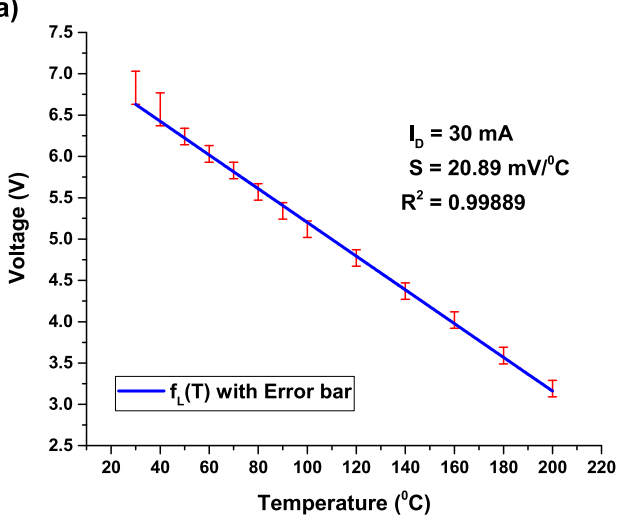

(b)

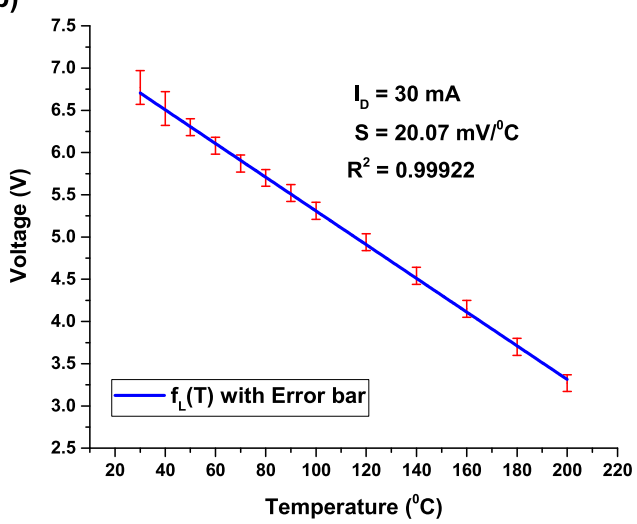

Fig. 6. Linear fit $f_{L}(T)$ with the Error bar of $V_{D}$ versus $T$, were characterized five times (a) $\mathrm{p}$-i-n configuration; (b) $\mathrm{p}$-i-n/ DWCNTs configuration (measurement cycles were done from $30^{\circ} \mathrm{C}$ to $200^{\circ} \mathrm{C}$ at a bias current $30 \mathrm{~mA})$.

this heat at the surface of the sensor. Therefore, measured linearity of the device is higher in case of DWCNTs version. With the data measured in the same figure, the best calculated linear pattern with a good degree of linearity is obtained. Calculated values of the coefficient of determination vary from 0.99883 up to 0.99182 (without DWCNTs) and from 0.99692 up to 0.99617 (a device introduced with DWCNTs) over the considered temperature range leading to a temperature sensing device were measured. The maximum value of $\mathrm{R}^{2}$ is 0.99922 for the modified device ( $\mathrm{p}-\mathrm{i}-\mathrm{n} / \mathrm{DWCNT}$ s configured) and 0.99889 for $\mathrm{p}-\mathrm{i}-\mathrm{n}$ configured occurs in the selected bias current range of 10-60 mA.
Sensitivity, $S$ of the sensor increases with the bias current in both cases, shown in Fig 5 (b). As a result, sensitivity varies from $17.39 \mathrm{mV} /{ }^{\circ} \mathrm{C}$ up to $20.06 \mathrm{mV} /{ }^{\circ} \mathrm{C}$ (in case of a device introduced with DWCNTs) and $18.03 \mathrm{mV} /{ }^{\circ} \mathrm{C}$ up to $22.34 \mathrm{mV} /{ }^{\circ} \mathrm{C}$ (without DWCNTs) occur in the bias current range of 10-60 $\mathrm{mA}$. Within the same current range, similar sensitivity variation stands at the same bias current points in both cases which indicates that no significant change in sensitivity has appeared, in case of DWCNTs presence too.

The Error analysis for sensing application is summarized in Fig 6 with the help of error bar, for selected bias current point $\mathrm{I}_{\mathrm{D}}=30 \mathrm{~mA}$ in both cases. The calculated maximum RMS error $\left(\mathrm{e}_{\mathrm{T}}\left[{ }^{\circ} \mathrm{C}\right]\right)$ values have been found lower than $\pm 2.85 \%$. In addition, the coefficient of determination is $\mathrm{R}^{2}=0.99889$, the corresponding sensitivity is $\mathrm{S}=20.89 \mathrm{mV} /{ }^{\circ} \mathrm{C}$ for $\mathrm{p}$-i-n configuration and $\mathrm{R}^{2}=0.99922$, the corresponding sensitivity is $\mathrm{S}=20.07 \mathrm{mV} /{ }^{\circ} \mathrm{C}$ for $\mathrm{p}$-i-n/DWCNTs configuration have been reported.

\section{CONCLUSION}

We have proposed, the comparative performance of two configurations of $\mathrm{p}-\mathrm{i}-\mathrm{n}$ and $\mathrm{p}-\mathrm{i}-\mathrm{n} / \mathrm{DWCNT}$ based temperature sensor in this work (sensors configurations were made of amorphous silicon and double walled carbon nanotubes). Analysis of sensitivity, $\mathrm{S}$ and coefficient of determination, $\mathrm{R}^{2}$ regarding temperature sensor have been measured for a device active area of $5.51 \times 5.77 \mathrm{~cm}^{2}$ in the biasing current range $\approx 10-60 \mathrm{~mA}$. The gained high sensitivity of sensor indicates that small change in temperature could be detectable. Many cycles of measurement were recapitulated five times diode showing excellent stability and output repeatability. The proposed amorphous silicon-based temperature sensor (introduced with DWCNTs) has been reported with excellent sensitivity, linearity and operating stability. These properties make it highly suitable to large surface area temperature sensing application.

\section{FUTURE DIRECTION}

Ability to be deposited on flexible substrate, makes a-Si very useful material to develop wearable devices in critical situation like dangerous radiation, charging of electronic devices, detection of IR rays etc. Thin film-based temperature sensor on flexible substrate can be useful for skin temperature measurement through wearable fabric. Moreover, attachment with 
athlete's kit would be more useful for real time temperature record and any other parameters like battery charging.

\section{REFERENCES}

[1] F. Morin, "Amorphous silicon TFTs and their applications," in Proc. 22nd Eur. Solid State Device Res. Conf. (ESSDERC), 1992, pp. 171-177.

[2] J. Henry and J. Livingstone, "Thin-film amorphous silicon positionsensitive detectors," Adv. Mater., vol. 13, nos. 12-13, pp. 1022-1026, Jul. 2001.

[3] G. de Cesare, A. Nascetti, and D. Caputo, "Amorphous silicon p-i-n structure acting as light and temperature sensor," Sensors, vol. 15, no. 6 , pp. 12260-12272, May 2015.

[4] T. Kamei, B. M. Paegel, J. R. Scherer, A. M. Skelley, R. A. Street, and R. A. Mathies, "Integrated hydrogenated amorphous Si photodiode detector for microfluidic bioanalytical devices," Anal. Chem., vol. 75, no. 20, pp. 5300-5305, Oct. 2003.

[5] D. Knipp et al., "Vertically integrated amorphous silicon color sensor arrays," IEEE Trans. Electron Devices, vol. 53, no. 7, pp. 1551-1558, Jul. 2006.

[6] R. A. Street et al., "High performance amorphous silicon image sensor arrays," J. Non-Crystalline Solids, vols. 227-230, pp. 1306-1310, May 1998.

[7] J. Magarino, "Amorphous silicon diodes and TFTs for active matrix flat panel display applications," Appl. Phys. A Solids Surf., vol. 41, no. 4, pp. 297-303, Dec. 1986.

[8] D. E. Carlson and C. R. Wronski, "Amorphous silicon solar cell," Appl. Phys. Lett., vol. 28, no. 11, pp. 671-673, 1976.

[9] X. Wang, Y. Han, and J. Si, "Non-uniformity emendation technique for amorphous silicon flat-panel detectors used for industrial X-ray digital radiography," Measurement, vol. 41, no. 7, pp. 817-822, Aug. 2008.

[10] R. A. Street, S. N. Kaplan, and V. Perez-Mendez, "High resolution amorphous silicon radiation detectors," Google Patents 5117114 A, May 26, 1992.

[11] J. A. Hidalgo-López, J. Romero-Sánchez, R. Fernández-Ramos, J. F. Martín-Canales, and J. F. Ríos-Gómez, "A low-cost, high-accuracy temperature sensor array," Measurement, vol. 125, pp. 425-431, Sep. 2018.

[12] S. K. Sen, T. K. Pan, and P. Ghosal, "An improved lead wire compensation technique for conventional four wire resistance temperature detectors (RTDs)," Measurement, vol. 44, no. 5, pp. 842-846, Jun. 2011.

[13] Y. Chen, H. Jiang, W. Zhao, W. Zhang, X. Liu, and S. Jiang, "Fabrication and calibration of $\mathrm{Pt}-10 \% \mathrm{Rh} / \mathrm{Pt}$ thin film thermocouples," Measurement, vol. 48, pp. 248-251, Feb. 2014.

[14] G. Liu, L. Guo, C. Liu, and Q. Wu, "Evaluation of different calibration equations for NTC thermistor applied to high-precision temperature measurement," Measurement, vol. 120, pp. 21-27, May 2018.

[15] M. Soni, M. Bhattacharjee, M. Ntagios, and R. Dahiya, "Printed temperature sensor based on PEDOT: PSS-graphene oxide composite," IEEE Sensors J., vol. 20, no. 14, pp. 7525-7531, Jul. 2020.

[16] Q. Liu, H. Tai, Z. Yuan, Y. Zhou, Y. Su, and Y. Jiang, "A high-performances flexible temperature sensor composed of polyethyleneimine/reduced graphene oxide bilayer for real-time monitoring," Adv. Mater. Technol., vol. 4, no. 3, Mar. 2019, Art. no. 1800594

[17] Z. Duan et al., "Facile, flexible, cost-saving, and environment-friendly paper-based humidity sensor for multifunctional applications," ACS Appl. Mater. Interfaces, vol. 11, no. 24, pp. 21840-21849, Jun. 2019.

[18] J. Joseph, S. G. Singh, and S. R. K. Vanjari, "Ultra-smooth e-beam evaporated amorphous silicon thin films-A viable alternative for PECVD amorphous silicon thin films for MEMS applications," Mater. Lett., vol. 197, pp. 52-55, Jun. 2017.

[19] N. Zhang, C.-M. Lin, D. G. Senesky, and A. P. Pisano, "Temperature sensor based on $4 \mathrm{H}$-silicon carbide $\mathrm{PN}$ diode operational from $20 \mathrm{C}$ to 600 C," Appl. Phys. Lett., vol. 104, no. 7, p. 73504, 2014.

[20] S. Pisana, S. K. O’Leary, and S. Zukotynski, "Optical properties of hydrogenated amorphous carbon thin films prepared by DC saddle field plasma-enhanced chemical vapor deposition," J. Non-Crystalline Solids, vol. 351, nos. 8-9, pp. 736-740, Apr. 2005.

[21] S. Paul and F. J. Clough, "A reliability of different metal contacts with amorphous carbon," Microelectron. Rel., vol. 42, no. 1, pp. 141-143, Jan. 2002

[22] F. W. Smith, "Optical constants of a hydrogenated amorphous carbon film," J. Appl. Phys., vol. 55, no. 3, pp. 764-771, Feb. 1984.

[23] R. Huang, Y. Cai, Y. Liu, W. Bai, Y. Kuang, and Y. Wang, "Resistive switching in organic memory devices for flexible applications," in Proc. IEEE Int. Symp. Circuits Syst. (ISCAS), Jun. 2014, pp. 838-841.
[24] V. Rojwal, M. K. Singha, T. K. Mondal, and D. Mondal, "Formation of micro structured doped and undoped hydrogenated silicon thin films," Superlattices Microstruct., vol. 124, pp. 201-217, Dec. 2018.

[25] B. Doherty, "PIN diode fundamentals," MicroNote Ser, vol. 701, pp. 1-2, 2006. [Online]. Available: http://www.microsemi.com

[26] F. Udrea, S. Santra, and J. W. Gardner, "CMOS temperature sensorsconcepts, state-of-the-art and prospects," in Proc. Int. Semiconductor Conf. (CAS), vol. 1, 2008, pp. 31-40.

[27] W. Shockley, "The theory of $p-n$ junctions in semiconductors and $p-n$ junction transistors," Bell Syst. Tech. J., vol. 28, no. 3, pp. 435-489, 1949.

[28] S. Santra, P. K. Guha, S. Z. Ali, I. Haneef, and F. Udrea, "Silicon on insulator diode temperature sensor-A detailed analysis for ultra-high temperature operation," IEEE Sensors J., vol. 10, no. 5, pp. 997-1003, May 2010.

[29] S. Rao, G. Pangallo, and F. G. D. Corte, " $4 \mathrm{H}-\mathrm{SiC}$ p-i-n diode as highly linear temperature sensor," IEEE Trans. Electron Devices, vol. 63, no. 1, pp. 414-418, Jan. 2016.

[30] N. J. D. Nagelkerke, "A note on a general definition of the coefficient of determination," Biometrika, vol. 78, no. 3, pp. 691-692, 1991.

[31] S. Rao, G. Pangallo, and F. Della Corte, "High perfomance integrated temperature sensor based on amorphous silicon diode for photonics on CMOS," in Proc. 4th Int. Conf. Photon., Opt. Laser Technol. (PHOTOPTICS), 2016, pp. 1-4.

[32] K. S. Karimov, F. A. Khalid, and M. T. S. Chani, "Carbon nanotubes based strain sensors," Measurement, vol. 45, no. 5, pp. 918-921, Jun. 2012

[33] S. Iijima, "Helical microtubules of graphitic carbon," Nature, vol. 354 , pp. 56-58, Nov. 1991.

[34] J. M. Planeix et al., "Application of carbon nanotubes as supports in heterogeneous catalysis," J. Amer. Chem. Soc., vol. 116, no. 17, pp. 7935-7936, Aug. 1994.

[35] R. Saito, M. Fujita, G. Dresselhaus, and M. S. Dresselhaus, "Electronic structure of chiral graphene tubules," Appl. Phys. Lett., vol. 60, no. 18, pp. 2204-2206, May 1992.

[36] P. Calvert, "Nanotube composites: A recipe for strength," Nature, vol. 399 , no. 6733 , p. 210,1999

[37] C. Q. Sun, H. L. Bai, B. K. Tay, S. Li, and E. Y. Jiang, "Dimension, strength, and chemical and thermal stability of a single $\mathrm{C}-\mathrm{C}$ bond in carbon nanotubes," J. Phys. Chem. B, vol. 107, no. 31, pp. 7544-7546, Aug. 2003.

[38] J. Park, M. F. P. Bifano, and V. Prakash, "Sensitivity of thermal conductivity of carbon nanotubes to defect concentrations and heattreatment," J. Appl. Phys., vol. 113, no. 3, p. 34312, 2013.

[39] C. Shen, A. H. Brozena, and Y. Wang, "Double-walled carbon nanotubes: Challenges and opportunities," Nanoscale, vol. 3, no. 2, pp. 503-518, 2011

[40] S.-Y. Yang et al., "Effect of functionalized carbon nanotubes on the thermal conductivity of epoxy composites," Carbon, vol. 48, no. 3 , pp. 592-603, Mar. 2010.

[41] J. Wang, H. Xie, Z. Xin, and Y. Li, "Increasing the thermal conductivity of palmitic acid by the addition of carbon nanotubes," Carbon, vol. 48 , no. 14 , pp. 3979-3986, Nov. 2010.

[42] M. F. L. De Volder, S. H. Tawfick, R. H. Baughman, and A. J. Hart, "Carbon nanotubes: Present and future commercial applications," Science, vol. 339, no. 6119, pp. 535-539, Feb. 2013.

[43] A. E. Aliev, M. H. Lima, E. M. Silverman, and R. H. Baughman, "Thermal conductivity of multi-walled carbon nanotube sheets: Radiation losses and quenching of phonon modes," Nanotechnology, vol. 21 no. 3, p. 35709, 2009.

[44] P. E. Hopkins and J. C. Duda, "Introduction to nanoscale thermal conduction," Heat Transf. Math. Model. Numer. Methods Inf. Technol, vol. 112, p. 872, Jan. 2006.

[45] C. W. Tan, K. H. Tan, Y. T. Ong, A. R. Mohamed, S. H. S. Zein, and S. H. Tan, "Energy and environmental applications of carbon nanotubes," Environ. Chem. Lett., vol. 10, no. 3, pp. 265-273, 2012.

[46] E. Pop, D. Mann, Q. Wang, K. Goodson, and H. Dai, "Thermal conductance of an individual single-wall carbon nanotube above room temperature," Nano Lett., vol. 6, no. 1, pp. 96-100, Jan. 2006.

[47] E. Thostenson, C. Li, and T. Chou, "Nanocomposites in context," Composites Sci. Technol., vol. 65, nos. 3-4, pp. 491-516, Mar. 2005.

[48] A. Jorio et al., "G-band resonant Raman study of 62 isolated singlewall carbon nanotubes," Phys. Rev. B, Condens. Matter, vol. 65, no. 15, Mar. 2002, Art. no. 155412.

[49] C. Thomsen and S. Reich, "Double resonant Raman scattering in graphite," Phys. Rev. Lett., vol. 85, no. 24, p. 5214, 2000. 
[50] R. R. Bacsa et al., "Narrow diameter double-wall carbon nanotubes: Synthesis, electron microscopy and inelastic light scattering," New J. Phys., vol. 5, no. 1, p. 131, 2003.

[51] U. D. Venkateswaran et al., "Probing the single-wall carbon nanotube bundle: Raman scattering under high pressure," Phys. Rev. B, Condens. Matter, vol. 59, no. 16, p. 10928, 1999.

[52] H. Kuzmany et al., "Determination of SWCNT diameters from the Raman response of the radial breathing mode," Eur. Phys. J. B-Condens. Matter Complex Syst., vol. 22, no. 3, pp. 307-320, Aug. 2001.

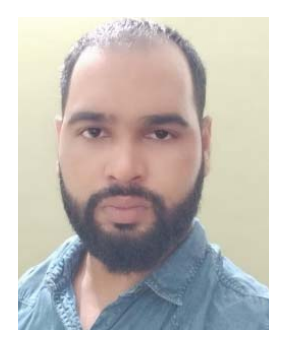

Vineet Rojwal is a Research Scholar with the Department of Instrumentation and Applied Physics, Indian Institute of Science, Bengaluru. He works on amorphous silicon (a-Si: H) thin films and investigated the connection between the plasma deposition conditions and properties of microcrystalline silicon $(\mu \mathrm{c}-\mathrm{Si}: \mathrm{H})$ for the various applications of optoelectronics. Moreover, he has developed and established a synthesis technique for carbon nanotubes in his laboratory, known as electric arc discharge. His keen interest is in nanostructures and thin films research in order to enhance the performance of devices, based on several applied physics principles such as photodetection, temperature, and gas sensing, as well as photocatalysis and photovoltaics.

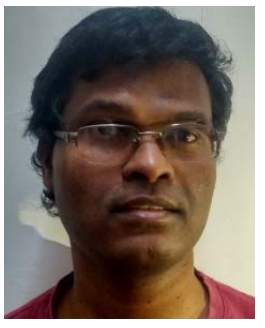

Monoj Kumar Singha is a Researcher with the Indian Institute of Science, where he is currently working on MEMS, nanotechnology, and microfluidics. He is developing a low-cost microstructure deposition technique for MEMS-based devices and sensors applications than the conventional deposition techniques like sputtering, thermal, and e-beam evaporation. He has expertise in MEMS, nanotechnology, and chemical sensors. He has published research papers on UV detector, photocatalysis, gas sensors, SERS, and solar cell absorber materials.

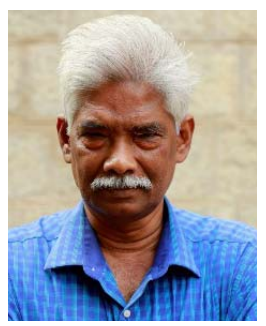

T. K. Mondal received the Ph.D. degree from the Indian Institute of Science, Bengaluru. $\mathrm{He}$ is currently a Principal Research Scientist with the Department of Instrumentation and Applied Physics, IISc Bengaluru. He has expertise in the high pressure, high-temperature system for electrical studies, and high-pressure differential thermal analysis system. Currently, his group is working on various applications of Applied Physics such as photovoltaics, carbon nanotube synthesis, and thin film-based gas sensors. 